\title{
Using Retrograde Flow from the Descending Genicular Artery: An Additional Safety Measure in Knee Coverage
}

\author{
Janna Joethy, MBBS, MRCS, MSc, FAMS ${ }^{1}$ Goh Jun Chance, MBBS, MRCS ${ }^{1}$ \\ Jeremy Sun Ming Fa, MBBS, MRCS, MMed ${ }^{1}$ Allen Wong, MBBS, MRCS, MMed ${ }^{1}$
}

Tan Bien-Keem, MBBS, FRCS, FAMS ${ }^{1}$

${ }^{1}$ Department of Plastic, Reconstructive and Aesthetic Surgery, Singapore General Hospital, Singapore, Singapore

J Reconstr Microsurg Open 2016;1:128-130.

\section{Introduction}

The knee joint possesses a rich anastomosis of blood vessels comprising of five major arteries. ${ }^{1}$ Aside from providing the knee with adequate blood supply, these vessels can be used in free flap surgery for anastomosis or as additional pedicles for added safety. We utilized retrograde flow via the descending genicular artery from the patellar arterial arcade as an additional safety measure for our free latissimus dorsi flap for knee wound coverage.

\section{Case Presentation and Methods}

A 50-year-old woman with no significant past medical history was admitted with a $1 \%$ full thickness burn over the anterior aspect of her knee. She underwent burns excision of the fullthickness burn tissue and negative pressure wound therapy. Intraoperatively, knee capsule was not breached and the patella, patella tendon, and paratendon were exposed.

After the wound debridement, the resultant defect measured 12 by $15 \mathrm{~cm}$. A free left latissimus dorsi flap was harvested based on the thoracodorsal vessels ( - Fig. 1). The branch to the serratus was identified and also included with the flap.

At the knee, an incision was made between the vastus medialis and sartorius. Dissection with loupes was performed until the descending genicular vessels were identified. The descending genicular artery was transacted at the hilt. The thoracodorsal artery was anastomosed in an end-to-side fashion to the femoral artery. The thoracodorsal vein was anastomosed end-to-end to the great saphenous vein. The flap was blanching well immediately post anastomosis but subsequently displayed poorer blood flow. Intraoperatively, we noted good flow and strong pressure head from the distal cut segment of the

received

June 6, 2016

accepted after revision

July 27, 2016

published online

September 14, 2016
Address for correspondence Goh Jun Chance, MBBS, MRCS, Department of Plastic, Reconstructive and Aesthetic Surgery, Singapore General Hospital, 20 College Road, Singapore 169856 (e-mail: chance_goh88@msn.com).

descending genicular artery and elected to anastomose this to the branch to the serratus anterior. The second venous anastomosis was performed end-to-end between the serratus branch vein and the descending genicular vein (-Fig. 2). Post anastomosis, good pulsation was seen with good flow to the flap and the flap was inset with no further events.

Postoperatively, the flap remained healthy. Color and turgor remained excellent during the rest of her admission. A split-thickness skin graft was placed over the muscle (See - Fig. 3). The patient was subsequently discharged after she was able to ambulate.

\section{Discussion}

\section{Difficulties in Knee Coverage}

The anterior knee is a difficult area to reconstruct despite its rich network of anastomoses. Coverage options are vast in number and range from conservative management, early skin grafting to flap surgery. In cases like ours, where the knee defect is large and deep with exposed vital structures, free flap surgery is recommended. ${ }^{2}$ Common free flap options are the latissimus dorsi flap, rectus abdominus muscle flap, anterolateral thigh flap, and free perforator flaps. Aside from difficulties in choosing the type of wound coverage, knee free flaps are reported to have a higher reexploration rate due to vascular thrombosis as compared with other free flaps. Depending on the literature used, the rate of knee free flaps requiring reexploration can double as compared with other free flaps $s^{3,4}$

\section{Why Latissimus Dorsi Flap}

The latissimus dorsi flap is a thin pliable muscle flap which we prefer over a bulky flap for good knee contour. Using a muscle flap has the added benefit of being more robust against
Copyright $\odot 2016$ by Thieme Medical Publishers, Inc., 333 Seventh Avenue, New York, NY 10001, USA. Tel: +1(212) 584-4662.

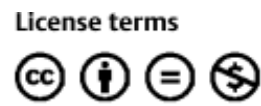

10.1055/s-0036-1592143 ISSN 2377-0813. 


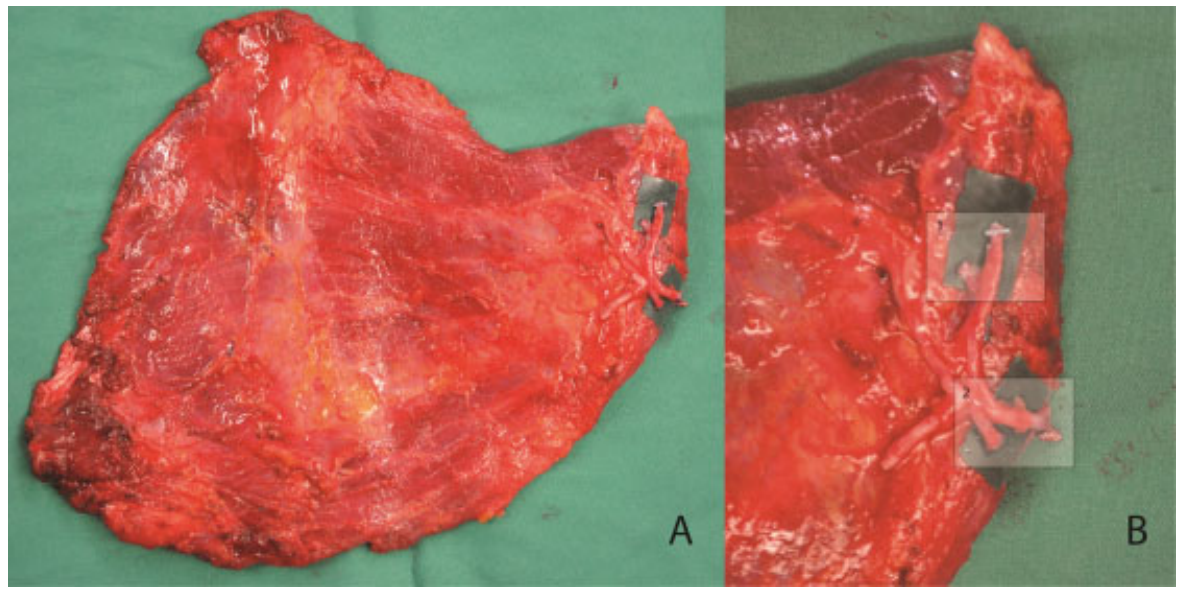

Fig. 1 (A-B) Intraoperative photo of the latissimus dorsi flap and a close-up of the pedicle. Box 1 showing the thoracodorsal artery and vein. Box 2 showing the branch to the serratus artery and vein.

infections. The latissimus dorsi flap was chosen in this case as the defect was large with exposed vital structures which required three-dimensional coverage and is robust against a contaminated wound. ${ }^{2}$ Limits of latissimus dorsi flap vascularity have been described previously and perfusion of latissimus dorsi flaps within the dimensions $15 \mathrm{~cm}$ long and $25 \mathrm{~cm}$ long are considered safe. ${ }^{5}$ Pedicled local flaps such as medial gastrocnemius flap were considered as well, but would not have been able to cover the entire defect.

\section{Anastomosis}

The need to improve blood supply arises from the potential complications more commonly associated with latissimus dorsi free flaps to the knee. Latissimus dorsi flaps have

\section{Diagram showing vessel layout and Anastamosis}

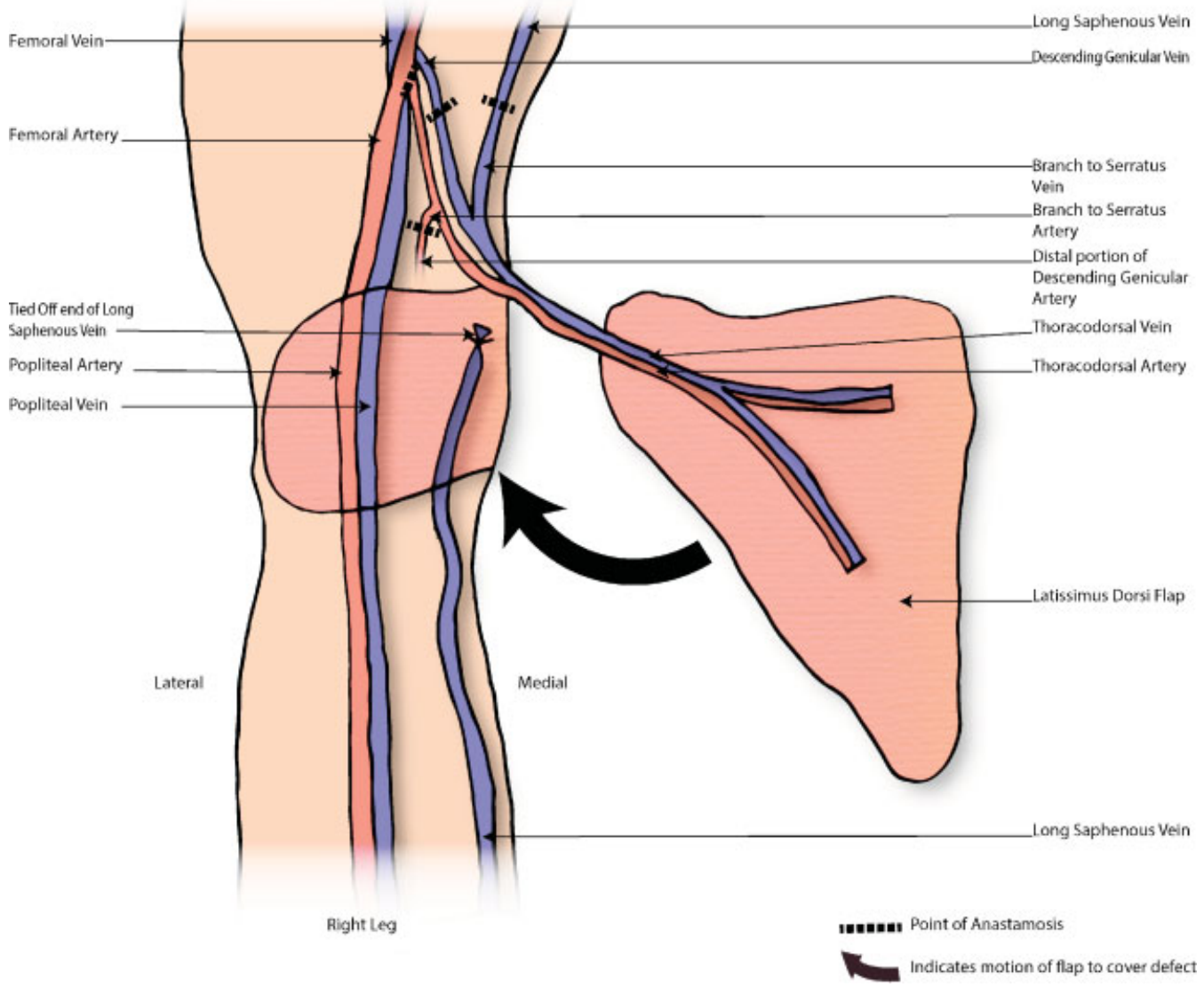

Fig. 2 Illustration showing the respective anastomoses. 


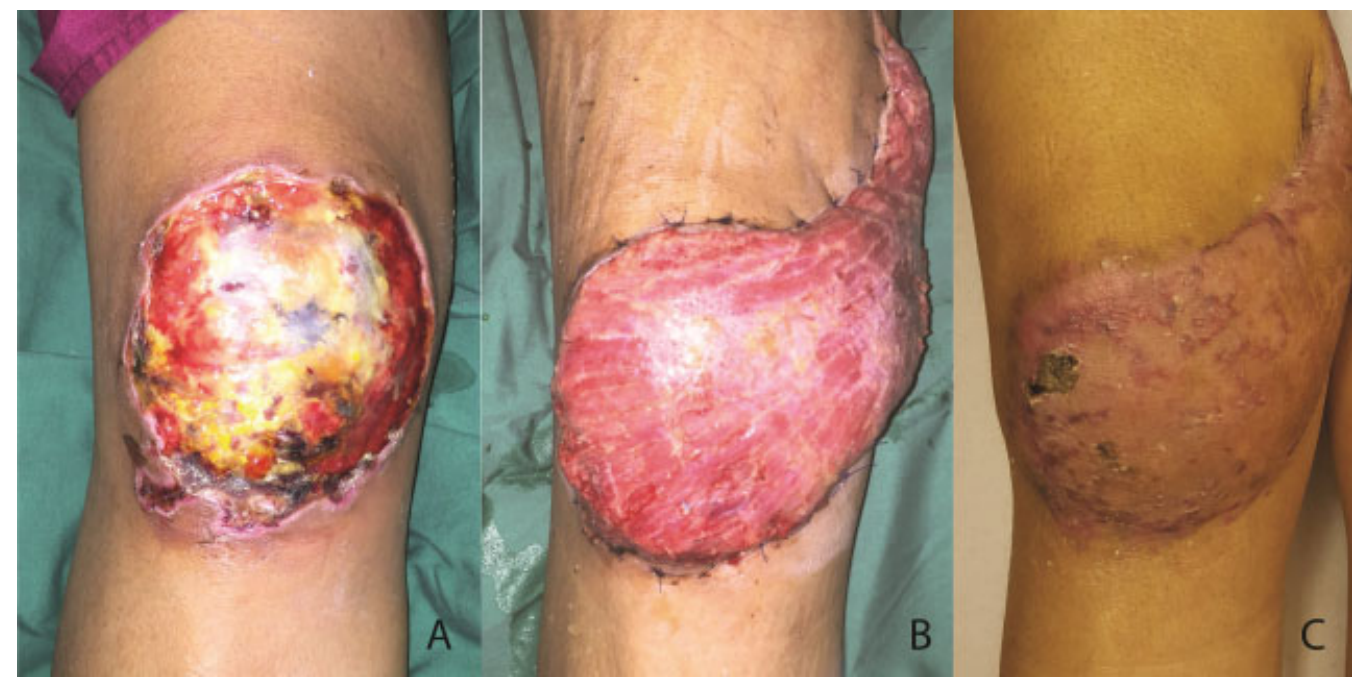

Fig. 3 (A) Preoperative photo of the wound. (B) Postoperative photo of the latissimus dorsi flap before skin grafting. (C) Follow-up photo in the clinic 2 months post operation.

been described to have a higher rate of flap failure as compared with that of other free flaps. ${ }^{4}$ Takagi et al described supercharging of the extended latissimus dorsi flap in breast reconstruction to help reduce the rate of partial flap necrosis by supercharging the latissimus dorsi flap via an intercostal branch. Bipedicled latissimus dorsi flaps have also been described to have decreased ischemia risk ${ }^{6}$ Our method of using retrograde flow from the distal cut end of the genicular artery aims to improve blood flow to the flap and provides an additional safety measure or salvage method for latissimus dorsi flaps with poor blood flow.

In our anastomosis, we used the cut distal portion of the descending genicular artery. We chose to use this vessel as it had a good pressure head intraoperatively. In anatomy textbooks, the descending branch of the lateral circumflex femoral artery is commonly depicted to have an anastomosis with a branch from the descending genicular artery, however, this anastomosis is only present in $30 \%$ of people. ${ }^{7}$ In our case, we expect the flow to be retrograde from this prepatellar arterial arcade.

\section{Conclusion}

Reconstruction of soft tissue over the knee joint is challenging. Using retrograde flow from the descending genicular artery is currently not commonly performed but should be considered as an additional safety measure as salvage for flaps with poor blood flow. Through this report, we hope to show the potential of retrograde flow from the descending genicular vessel of the knee as a site for additional anastomo- sis used for wound coverage of the knee. We believe our case to be the first presentation of using retrograde flow from the descending genicular artery for anastomosis of a free latissimus dorsi flap in the knee based on Pubmed, Medline, and Embase literature search using the keywords "retrograde flow," "latissimus dorsi free flap", and "knee wound."

\section{References}

1 Shim SS, Leung G. Blood supply of the knee joint. A microangiographic study in children and adults. Clin Orthop Relat Res 1986; (208):119-125

2 Gravvanis A, Kyriakopoulos A, Kateros K, Tsoutsos D. Flap reconstruction of the knee: a review of current concepts and a proposed algorithm. World J Orthod 2014;5(5):603-613

3 Louer CR, Garcia RM, Earle SA, Hollenbeck ST, Erdmann D, Levin LS. Free flap reconstruction of the knee: an outcome study of 34 cases. Ann Plast Surg 2015;74(1):57-63

4 Khouri RK, Cooley BC, Kunselman AR, et al. A prospective study of microvascular free-flap surgery and outcome. Plast Reconstr Surg 1998;102(3):711-721

5 Karaaltin MV, Erdem A, Canter I, Cavdar G, Baghaki S. The bipedicled latissimus dorsi myocutaneous free flap: clinical experience with 53 patients. Microsurgery 2010;30(3):179-184

6 Takagi S, Oyama T, Yamazumi K, Eto A, Ohjimi H. Vascular augmentation of an extended latissimus dorsi myocutaneous flap through an intercostal vessel: a preliminary report. J Plast Surg Hand Surg 2013;47(2):123-125

7 Sabalbal M, Johnson M, McAlister V. Absence of the genicular arterial anastomosis as generally depicted in textbooks. Ann R Coll Surg Engl 2013;95(6):405-409 